\title{
Editorial
}

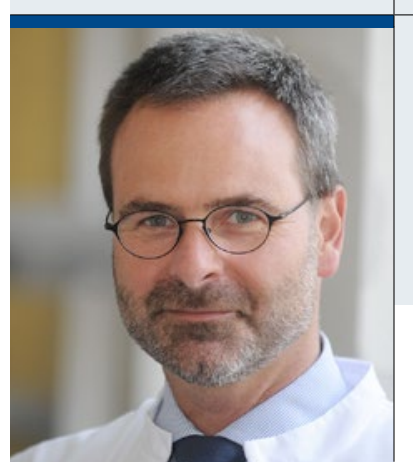

"In der Therapie von Hodentumoren geht es inzwischen nicht

mehr nur allein um die Heilung, sondern um die Heilung ohne

Langzeitfolgen."

Univ.-Prof. Dr. med. Peter Albers

Direktor der Klinik für Urologie, Universitätsklinikum Düsseldorf, Medizinische Fakultät der Heinrich-Heine-Universität Düsseldorf

\section{Der Hodentumor - selten und gefährlich!}

E in Allgemeinarzt sieht im Durchschnitt einen Hodentumorpatienten in 24,1 Jahren Praxistätigkeit, $30 \%$ der histopathologischen Befunde sind nicht korrekt, initiale Therapievorschläge treffen auch bei Fachärzten zu 30 \% nicht zu, das Überleben nach Behandlung in einem Zentrum für Hodentumoren ist $10 \%$ besser und das Risiko an einem komplexen Hodentumor zu versterben außerhalb eines solchen Zentrums doppelt so hoch: Warum also glauben wir immer noch als Ärzte alles und jedes gleich gut zu können?

\section{Heilung ohne Langzeitfolgen}

Die 2015 publizierte EUROCARE-5-Studie zeigt, dass Deutschland in der Analyse der 5-Jahres-Überlebensrate von Hodentumoren unter den 14 Ländern in Nord- und Zentraleuropa nur auf Platz neun liegt - bei einem Tumor, der üblicherweise zu weit über $90 \%$ stadienunabhängig geheilt wird! In die Überlebensdaten gehen die durch eine Salvage-Therapie letztlich doch noch geretteten Patienten nicht ein.

30 Jahre nach Chemotherapie oder Bestrahlung eines Hodentumors liegt die Herzinfarktrate des Patienten beim Doppelten des Üblichen. Die Rate an therapieinduzierten Zweitmalignomen steigt zum Teil auf das Dreifache. Daher geht es in der Therapie von Hodentumoren inzwischen nicht mehr nur allein um die Heilung, sondern um die Heilung ohne Langzeitfolgen!

Die in dieser Ausgabe dargestellten aktuellen Therapierichtlinien und neuen Daten zur Lebensqualität nach Therapie geben sehr überzeugend einen Überblick über die inzwischen extrem stadienspezifische, differenzierte Behandlung, die vor allem das Ziel hat, nur dann toxisch zu sein, wenn es unbedingt erforderlich ist.
Im Interesse der uns anvertrauten jungen Patienten mit Hodentumoren sollte jeder von uns daran denken, dass diese Patienten üblicherweise eine uneingeschränkte Lebenszeit haben und daher alle Langzeittoxizitäten der Therapie erleben werden. Dies ist fundamental anders als bei Blasenkarzinomoder Prostatakarzinompatienten. Lebensqualität bedeutet das uneingeschränkte Gehör des Musikers, das erhaltene Feingefühl des Goldschmieds oder eben auch die uneingeschränkte Zeugungsfähigkeit des Familienvaters.

\section{Die Therapie muss zentralisiert werden}

Bevor dogmatisch und schablonenartig adjuvant im Frühstadium chemotherapiert wird, bevor aus Unkenntnis eine Residualtumorresektion zur retrograden Ejakulation führt oder bevor gar ein Zyklus Salvage-Chemotherapie zu wenig zur Hochdosis-Rescue zwingt, sollte jeder Arzt die Möglichkeit einer Zweitmeinung über das Zweitmeinungsportal (www.zm-hodentumor.de) nutzen oder den Patienten in einem spezialisierten Zentrum (www.hodenkrebs.de) vorstellen. Andere Länder haben uns vorgemacht, wie wir vorgehen sollten: durch Zentralisierung der Therapie. Seltenes ist selten. Wir sollten wie bei allen Entscheidungen unseres Berufs die Verantwortlichkeit für das Schicksal des Patienten an erste Stelle setzen.

Viel Freude bei der Lektüre dieser Ausgabe der URO-NEWS wünscht mit kollegialen Grüßen,

Ihr

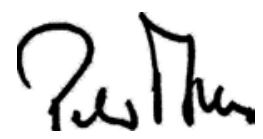

